\title{
A experiência dos clássicos adaptados: caminhos ínvios na promoção da leitura de textos literários?
}

The experience of adapting the classics:

narrow paths in the promotion of literary readingy

\author{
PAULO COSTA \\ Universidade de Évora \\ Portugal \\ paulo.lampreia.costa@gmail.com
}

Resumo. A adaptação de obras consideradas como "clássicos da literatura portuguesa", não sendo uma prática nem recente nem inovadora tem, desde há algum tempo, conhecido novos contornos no mercado editorial nacional. Procuraremos, com a presente reflexão, colocar criticamente em destaque alguns aspectos: a) algumas reflexões teóricas em torno do texto literário e do cânone; b) leitura de textos literários e funções que aquela pode desempenhar c) características e questões de natureza geral levantadas pelas adaptações de textos literários não direccionados inicialmente para uma recepção infantil e/ou juvenil (a quem se destinam efectivamente estes textos?).

Tomando como objecto de análise alguns exemplos recentemente publicados, tentaremos questionar os aspectos anteriormente referidos. Neste percuso, procuraremos ver em que medida se visa 0 melhor de dois mundos: o das crianças e jovens, o dos pais e educadores, o do nicho de mercado eventualmente ainda por explorar. Talvez o 'mapa' nos guie até uma 'Terra do Nunca' na qual uma aparência de produto pedagógico ou similar sirva prioritariamente propósitos comerciais.

Palavras chave: adaptação dos clássicos; educação literária; cânone; literatura portuguesa para crianças.
Abstract. Adapting literary texts, considered either "classics of Portuguese literature", or as the work of renowned authors is a kind of enterprise that cannot be considered recent or innovative. However, in recent times, new features have emerged as new texts arrive on the market through the hands of publishers. In this paper, we will try to take a critical look at the following aspects: a) general implications of the process of adapting to children, texts that, in their genesis, were not directed to this kind of readership ; b) the objectives which actually guide the production of these texts; c) who the authentic target of these texts is.

After selecting some examples of recently published books we will present some reflections on the topics referred to in the previous paragraph. We hope that if we cannot provide the answers, we are able to, at least, perceive if those texts aim for the best of both worlds: the one of children and young adults and the one of parents (and teachers), specific and always renewable commercial targets. Maybe the 'map' will lead us to a 'neverland' where pedagogic appearances hide and serve purely commercial purposes.

Key words: classics adaptation; literary education; canon; Portuguese children literature.

\footnotetext{
I Para citar este artículo: Costa, P., A experiência dos clássicos adaptados: caminhos ínvios na promoção da leitura de textos literários? Alabe 4, diciembre 2OII [http://www.ual.es/alabe]
} 


\section{Introdução: algumas reflexões teóricas em torno do texto literário e do cânone}

A existência de iniciativas, de natureza distinta, fruto de motivações de diversa ordem, visando o contacto de uma determinada faixa etária de leitores, abrangendo crianças e jovens, tendo como ponto de partida obras literárias, relativamente às quais parece haver o consenso de que teriam como destinatário imediato um leitor adulto, não constitui uma novidade em Portugal. Não será objectivo deste texto a reconstituição desse percurso na diacronia; procuraremos, sobretudo, reflectir sobre alguns dos aspectos que, no plano teórico são, em nossa opinião, passíveis de serem equacionados, recorrendo para tal, à caracterização de uma das recentes iniciativas do mercado editorial em Portugal neste âmbito. Assim, reflectindo sobre aspectos de natureza mais abrangente levantados pela produção e divulgação de textos deste teor: os textos em análise constituem os quatro volumes integrantes da colecção "Clássicos a brincar”, de Sara Rodrigues (texto) e Cristiana Resina (ilustração), editados pelas Edições Asa, até Julho de 2OIr. Procuraremos sistematizar algumas considerações, quer de natureza teórica, quer a partir da análise dos textos que mencionámos, centradas nas seguintes linhas orientadoras: a) algumas reflexões teóricas em torno do texto literário e do cânone; b) leitura de textos literários e funções que aquela pode desempenhar c) características e questões de natureza geral levantadas pelas adaptações de textos literários não direccionados inicialmente para uma recepção infantil e/ou juvenil (a quem se destinam efectivamente estes textos?).

Poderemos, pelo que de comum apresenta esse 'escrever de outra forma', a atribuição de novo significante para, supostamente, um mesmo significado, comparar o exercício de adaptação de um texto ao processo de tradução, sobretudo se mediado por ferramentas informáticas que possam distender ainda mais o espaço entre o material originalmente escrito e o leitor. Ao afirmar que "Os programas de tradução automática são animais primitivos, perfeitamente incapazes de se orientarem no meio da pluralidade semântica dos significantes e do contexto informativo, já de si rico de significado na linguagem natural, quanto mais na linguagem literária [...]”, Steiner (2007) remete-nos para as questões da interpretação e para a sua complexidade. De facto, e forçando um pouco esta aproximação, uma adaptação, tal como uma tradução (sobretudo se literal ou mediada por software pouco espacializado) será, em última instância, um 'escrever de novo' e, consequentemente, não será de todo abusivo que possamos passar a falar de outro objecto textual, autónomo. Aliás, o próprio texto literário, enquanto objecto de características tipificáveis e passíveis de o identificarem enquanto tal, revela-se enquanto objecto cuja natureza carece de discussão profunda. Não sendo objectivo prioritário desta reflexão, julgamos pertinente a apresentação de algumas posições que possam apresentar o texto literário como objecto de contornos nem sempre consensuais. Assim, poderíamos caracterizar o texto literário no seguinte sentido:

Literature [...] is a speech act or textual event that elicits certain kinds of attention. Is contrasts with other sorts of speech acts, such as imparting information, asking questions, or making pro- 
mises. Most of the time, what leads readers to treat something as literature is that they find it in a context that identifies it as literature [...]. (Culler, 2000, p. 27)

De forma mais radical, poderíamos descrever assim a mesma realidade:

In analogy with the dynamic composition of the universe, I shall designate as dark matter the domain of immanent plurality that takes literature beyond the referential determinations of language or, put another way, that makes literature paradoxically exist as a continuous representation of a wordless experience of things. (Martins, 2003, p.r93)

Para além da complexidade do objecto texto literário/'literatura’, e uma vez que a adaptação de clássicos se configura aqui como o centro das nossas atenções, importará ainda que reflictamos sobre aquilo que poderemos designar como 'clássico’, no sentido de podermos compreender os critérios que terão presidido à escolha dos originais a adaptar por parte da autora/editora da colecção em análise neste texto.

Se o primeiro critério para a identificação de um clássico puder ser a de integrar o corpus composto por aqueles objectos que, pelas suas características intrínsecas ou reconhecidas exogenamente, poderão, neste âmbito, atingir ou não o estatuto de canónico, não estamos perante uma realidade isenta de polémica. Tal poderia levar-nos a admitir, num primeiro momento, que no epicentro dessa polemização estariam exactamente categorias/instâncias como os textos e/ou os autores ${ }^{2}$. Contudo, nem sempre o protagonismo é conferido a essas entidades, sendo o fulcro desta questão, por vezes apenas agitada, muitas vezes fracturante, transferido, na quase totalidade dos casos, para aqueles que reflectem sobre o fenómeno e/ou para os locais de génese ou de acolhimento dessa mesma reflexão, nomeadamente no plano académico, ou para os lugares nos quais determinadas escolhas fazem sentir claramente o seu efeito, com é o caso do contexto educativo. Em qualquer dos casos, a pedra de toque, neste problema, tenderá a identificar-se com a instância de validação da canonicidade o que, dadas as dificuldades de corporização de tal instância, não apenas dificulta a clarificação do problema como o contém e enforma. Traçando em breves linhas o percurso do cânone, torna-se evidente que, desde a Antiguidade, um carácter de relativa normatividade é associado ao termo. Etimologicamente, cânone, do grego kanon, remete-nos, de forma mais imediata, para sentidos muito concretos como barra de madeira comprida e direita, ou ainda régua, fiel da balança, entre outros. Em sentido figurado, o termo poderia então referir-se a regra, modelo, princípio e, aplicado à gramática, poderia abranger os sentidos de regra, modelo de declinação $e$ conjugação ou regra de gramática. Por via do latim canon, ter-nos-iam chegado, como sentidos mais relevantes neste contexto, os que reforçam essa ideia de lei, regra, medida, bem como a referência ao conjunto de textos/livros aos quais a Igreja reconheceria o estatuto decorrente de resultarem de inspiração divina. Nesta acepção de regra de gramática, Machado (I990) apresenta, a título de exemplo, o "cânone alexandrino", entendido

${ }^{2}$ Veja-se, a este respeito, a forma quase dogmática como Lleal (2OIO) se refere a esta ligação entre cãnone e clássico: "[...] es que algú pot dubtar que un clàssic, és a dir, una obra que mereixi formar part del cànon [...]?” (p. IO) 
como uma “[...] lista, organizada pelos gramáticos alexandrinos, dos melhores clássicos gregos" [...]”(p.55) Assim, interessar-nos-á uma perspectiva segundo a qual um determinado objecto, nomeadamente aqueles que classificaríamos como de natureza textual, em função das condições existentes, em determinado momento, para determinada comunidade, no que se refere aos materiais, à tecnologia e às técnicas disponíveis e, consequentemente, às suas condições de circulação, poderá desempenhar funções que o coloquem em vantagem relativamente a outros, do ponto de vista da sua sobrevivência. Entendemos que a valorização das funções que pode desempenhar, funções essas que, por seu turno, poderão conjunturalmente flutuar no plano diacrónico, será mais eficaz no desencadear de mecanismos de obtenção e/ou manutenção da canonicidade de um determinado texto ou conjunto de textos do que as suas próprias características. Neste sentido "[...] what may be spoken of as the "properties" of the work - its "structure," "features," "qualities," and, of course, its "meanings" - are not fixed, given, or inherent in the work "itself" but are at every point the variable products of some subject's interaction with it." (Smith, 2OOO, p.I48) Parece-nos fundamental a opção por um entendimento do cânone enquanto capital cultural e, portanto, numa perspectiva que equacione a importância dos factores de natureza humana, cultural, social e ideológica, sem excluir a eventualidade de o potencial intrínseco de determinados textos poder configurar-se ou ter-se configurado como relevante. Assim,

"If we grant a need for this memory and the manifest power of various canonical works to transcend any particular structure of social interest, it becomes possible to recuperate for our culture some of the values attributed in the past to traditional ideas of a high canon." (Altieri, I99O, pp. 22-23)

Said (2000), ainda a este propósito, afirma que "[...] while it is not necessary to regard every reading or interpretation of a text as the moral equivalent of a war or a political crisis, it does seem to me to be important to underline the fact that whatever else they are, works of literature are not merely texts." (pp. I98-199) Acrescenta o mesmo autor que "[...] during the many harangues on both sides of the so-called Western canon debate [...] so many of the combatents have ears of tin, and are unable to distinguish good writting and politically correct attitudes [...].”(p. 199 ${ }^{3}$

No que respeita à constituição do cânone, bem como em relação a outras construções que a história e a cultura vão integrando, e relativamente às quais parece desenvolver-se uma espécie de compulsão para a rejeição ou aceitação incondicionais, parecem-nos pertinentes as palavras com que Said (I994) inicia um dos seus textos:

Appeals to the past are among the commonest of strategies in interpreting the present. What animates such appeals is not only disagreement about what happened in the past, but uncertainty about whether the past really is past, over and concluded, or whether it continues, albeit in di-

3 Para uma visão sintética e crítica do trabalho de Said e do seu conceito de ‘open-canon’, veja-se Gorak (20OI, pp. I86-220) 
fferent forms, perhaps. This problem animates all sorts of discussions - about influence, about blame and judgement, about present actualities and future priorities. (p. 3 )

Não se tratará tanto de encontrar verdades absolutas como de permitir uma reflexão que possa integrar um entendimento dos textos literários, bem como outros elementos daquilo que consideraríamos como capital cultural, aqui entendido no sentido muito restrito em que Bourdieu (I993) o define, como conjunto de produtos artísticos de características particulares cujos mecanismos de produção, difusão, circulação e aceitação enquanto susceptíveis de desempenhar funções relevantes, não seriam auto-suficientes, antes seriam dependentes de condições particulares e de regras que regulariam as suas condições de existência, estatuto e funções ${ }^{4}$. A problemática dos clássicos, daqueles que, emergindo de entre os eleitos do cânone assumem um estatuto particular que os leve a serem classificados enquanto tal, será discutida, neste texto, num momento mais próximo do da caracterização da colecção Clássicos a brincar.

\section{A leitura: os textos e as funções que estes podem desempenhar}

A edição de textos destinados, formalmente, a um consumidor indeterminado, mas focada no possível estabelecimento de uma relação forte entre esse livro/produto e a leitura de textos similares, aproximáveis, com presença nos planos de estudos oficiais, em contexto escolar, implica que reflictamos sobre a leitura enquanto prática escolarizada. Poderemos partir do pressuposto de que, para um aluno cuja meta pessoal, no plano do investimento aplicado na sua formação, apontasse, no mínimo, para a conclusão da escolaridade obrigatória, um percurso com sucesso pelos diferentes níveis de ensino possibilitar-lhe-ia a prática da leitura com um nível progressivamente mais elaborado de proficiência. Este raciocínio implicaria igualmente que quanto mais baixo o grau de escolarização, mais baixo seria o nível de proficiência na leitura. Ao perspectivarmos estas considerações, estamos a assumir que, de entre as funções da leitura e, particularmente da escola enquanto espaço de promoção da leitura, surgiriam como fundamentais aquelas que classificaríamos como de âmbito social. Em textos diversos, encontramos reflexões neste sentido, discutindo as implicações de um entendimento da leitura como prática ou conjunto de práticas susceptível de desempenhar funções sociais de natureza diversa. Estas funções estariam intimamente ligadas a um entendimento da leitura como uma

\footnotetext{
${ }^{4}$ Bourdieu (I993) considera a cultura enquanto "[...] system of schemes of perception, expression and historically conditioned thinking[...]." (p. 233) Os diversos objectos que circulam neste âmbito constituirão o capital cultural, sendo a escola uma das instituiçôes que, de forma mais evidente, possibilitará a acumulação desse capital. Nesta linha, veja-se o texto de During (2000), no qual se afirma que, perante o final da literatura inglesa enquanto disciplina escolar, aquilo que se ensina é cultura, numa defesa radical dos estudos culturais e das suas funções/vantagens perante um cenário de globalização irreversível.
} 
prática susceptível de induzir benefícios, mais ou menos evidentes, no indivíduo, com consequências, supostamente positivas na sua qualidade de vida ${ }^{5}$.

Esta problemática estaria igualmente ligada às questões que se prendem com as categorias literacia e iliteracia, sendo que, ao admitirmos aquelas como válidas, estamos a fazer-lhes, imediatamente, corresponder as categorias letrado e iletrado ${ }^{6}$. Tais pressupostos remetem-nos para a ideia de que existirá um limiar entre categorias e, por sua vez, uma espécie de "mínimos" exigíveis para que aquele possa ser franqueado. Por outro lado, avança-se, ao nível do senso comum, com considerações que apontarão para um decréscimo do número de leitores, uma degradação do conjunto de práticas que se aceitou ser englobável na categoria 'hábitos de leitura'. Na prática, muitas vezes, estes discursos seriam, acima de tudo aquilo a que Dionísio (2000) se refere enquanto vozes apocalípticas, no mesmo sentido em que o fazem Chartier \& Hébrard (2000) ao questionar aquilo que designam de discursos públicos escandalizados. Em face dos números, quer o discurso dos media, quer o do poder político, tenderão a tomar uma atitude reactiva em função da forma como a opinião pública tendencialmente se manifesta. Disso será um exemplo claro o conjunto de textos publicados nos media em momentos subsequentes à divulgação de resultados de testes como os PISA. Considerando-se que a leitura será um claro indicador dos resultados (referidos como maus/péssimos) de que o investimento a que o tecido social procedeu, no sentido de que as gerações escolarizadas pudessem constituir-se como gerações de sucesso, independentemente dos parâmetros pelos quais este se possa aferir, a reacção é inevitável. Tenderão os media a enfatizar os aspectos mais negativos provocando, no plano político a emergência de medidas (supostamente) correctivas.

Uma outra possibilidade, com potencial de mercado muito evidente, é a escolha, por parte de determinados autores, quer aqueles tradicionalmente ligados ao campo literário, quer aqueles que têm vindo a marcar mais recentemente, presença no mercado editorial, por textos que, fazendo parte, em modalidades diversas e com estatutos e funções distintas no plano das opções curriculares, do grupo de textos com os quais os alunos, ao longo da escolaridade obrigatória terão de/deverão interagir, enquanto objectos passíveis de manobras de reescrita. $\mathrm{O}$ objectivo prioritário seria a reconfiguração desses textos, quer relativamente ao tipo de leitor a que se destina, quer, em alguns casos, enquanto objectos textuais diversos. Adaptar, como referimos anteriormente, pode não corresponder a um reescrever mas, sobretudo, a um escrever algo de diverso. A distância

\footnotetext{
5 Entende-se aqui a expressão qualidade de vida enquanto abstracção que possa conter um conjunto de condições nos planos material, cognitivo, intelectual, afectivo, isto é, cobrindo todas as vertentes da vida do indivíduo em sociedade e que correspondam, no mínimo, às expectativas que para si próprio e para o contexto que o envolve, foram criadas, no sentido de permitir um percurso de vida perspectivado pelo próprio enquanto factor de garante da sua dignidade nos planos pessoal e social. Consideramos assim que um incremento da qualidade de vida corresponderia a atingir ou superar essas expectativas.

${ }^{6}$ Entendemos aqui literacia num sentido mais abrangente do que o domínio de capacidades automáticas e universalmente aplicáveis, independentemente do contexto em que são activadas. "A literacia é [...], mais do que um conjunto de saberes, uma prática." (Dionísio, 2OOO, p. 24) Veja-se também o conjunto de textos compilados por Cassany (2009) explorando as múltiplas possibilidades de actualização deste ‘ser-se letrado’.
} 
entre o leitor e uma ideia que do texto original se tem é/pode aparentemente ser encurtada, embora, na prática se possa distanciar significativamente o leitor do texto original, nesse processo de ilusão adaptativa.

\section{A colecção 'Brincar aos clássicos': estratégias de adaptação}

A produção de versões de ‘clássicos para crianças’, ou a produção de adaptações, aceitando este termo como passível de englobar o conjunto de procedimentos/estratégias de reescrita e de resultados desse processo, não é, como mencionámos no início deste texto, algo de original no panorama editorial contemporâneo, em Portugal. Há, em Portugal, diversas colecções deste tipo de textos, de diversos períodos históricos, consistindo o fenómeno mais recente num ressurgimento deste tipo de iniciativas. Da mesma forma, não se trata de uma novidade no plano da investigação em torno do texto literário e/ou da investigação no campo educacional.

Pensamos que a variação no entendimento das funções que a escola, enquanto instância de institucionalização do conhecimento, poderá desempenhar, influencia a importância que determinados objectos poderão adquirir se forem vistos como potenciadores daquilo que os pais/encarregados de educação percebem como sucesso. O primeiro número da colecção apresenta, aliás, ao nível dos elementos paratextuais, o conjunto de características desta iniciativa editorial, destacando o mérito dos originais mas, sobretudo, o seu potencial propedêutico.

Relativamente à noção de clássico, elemento caracterizador dos textos eleitos nesta proposta editorial como pontos de partida, poderíamos apontar, discutindo esta classificação, que

[...] a "classic" is the product neither of the objectively (in the Marxist sense) conspiratorial force of establishment institutions nor of the continuous appreciation of the timeless virtues of a fixed object by succeeding generations of isolated readers, but rather, of a series of continuous interactions among a variably constituted object, emergent conditions, and mechanisms of cultural selection and transmission. (Smith, 2000, pp. I47-I48)

Por outro lado,

The classics are those books which constitute a treasured experience for those who have read and loved them; but they remain just as rich an experience for those who reserve the chance to read them for when they are in the best condition to enjoy them.” Calvino (2000, pp. 4)

Não poderemos deixar de considerar que a vulgarização do termo poderá ter-lhe conferido uma maior abrangência semântica, continuando a ser marcante a noção do texto que suporta ou se presta de forma particular à releitura, do texto que assume esse estatuto patrimonial que implicará a impossibilidade de que as gerações futuras possam ser privadas do seu contacto. Sabemos que não deixam as sociedades ao acaso o modo como 
essa transmissão é feita. As questões em torno do cânone, nomeadamente do cânone literário escolar, são uma das facetas emergentes do modo como as opções de natureza curricular, entendendo o currículo como construção social, se revelam como evidências desse mandato passado pelas comunidades, em momentos específicos, à Escola enquanto instituição.

Constituem esta colecção os seguintes textos: Os Miaus (2007) [Ti], Auto da Barca do Castigo (2008) [T2], Eurico, o Insecto (2009) [T3], Sermão dos Peixes aos Meninos (2OIO) [T4], da autoria de Sara Rodrigues, com ilustrações de Cristiana Resina, sob a chancela das Edições ASA. Os hipotextos correspondem ao seguinte conjunto de obras: Os Maias, de Eça de Queirós; Auto da Barca do Inferno, de Gil Vicente; Eurico, o Presbítero, de Alexandre Herculano; Sermão de santo António aos Peixes, de Padre António Vieira. As edições originais destes textos datam de I888, I5I7, I844, I654, respectivamente.

Ao analisar estas reescritas, propomo-nos, sobretudo, descrever as estratégias que terão presidido a esta re-produção/re-criação dos textos, não deixando de assumir que um dos nossos objectivos será a possibilidade de levantar questões que possam, num plano mais abrangente que o do presente artigo, colocar-nos na rota da reflexão sobre se este 'adaptar os clássicos’ poderá pretender corresponder a:

- permitir às crianças o contacto com obras de difícil acesso, quer pela sua complexidade estrutural e/ou linguística, quer pelas especificidades decorrentes dos seus contextos de produção?

- converter estes textos, assumidos como literário e como clássicos da literatura Portuguesa, em textos de 'leitura fácil'?

- tentar que as crianças leiam, num momento específico, textos que poderiam ler e valorizar mais tarde?

- permitir às crianças um alargamento das suas referências culturais (correspondendo a leitura a uma rarefação do do texto literário, restringindo-o ao factual)?

- reduzir o nível de ansiedade dos adultos que entendem ser necessário apressar o crescimento das crianças?

- realizar um bom investimento no plano editorial?

- possibilitar um bom investimento no plano curricular ('livros que um dia farão parte do currículo')?

- escolarizar os 'clássicos’?

\section{Estratégias de adaptação: alguns destaques}

No que se refere à estrutura formal, podemos considerar que, na generalidade, há uma preocupação por parte da autora? ${ }^{7}$ em manter as características gerais que, de forma

\footnotetext{
7 “Não integraremos neste texto a análise do texto icónico.
} 
mais ou menos consensualizada, no plano dos estudos literários, seriam identificadoras do género em questão. O exemplo mais evidente será o de T4, dado que o modelo que, no plano da retórica o sermão constitui, seria um constrangimento difícil de ignorar.

Por outro lado, apesar de os textos originais terem características e volumes muito diversos, as reescritas apresentam um volume muito semelhante, o que se ligará, de forma mais concreta com possíveis exigências editoriais do que com uma estratégia ou conjunto de estratégias concretas de adaptação de texto (TI-pp. 76; T2-pp. ıo7; T3-pp. 9I; T4-pp.8o). Os textos narrativos são objecto de uma enorme contracção, uma vez que os textos são originalmente longos; paradoxalmente o texto dramático, sendo originalmente o mais curto, é o mais longo desta colecção, o que contribui para esta ideia da tipificação dos diversos volumes da colecção.

Todos os textos seguem, em geral, uma opção reconhecível, pelo leitor comum, como semelhante à da fábula, pela invariável conversão dos humanos em animais. Neste processo são utilizadas diversas estratégias:

- $\quad$ Em Tı, segue-se, de perto, a proximidade entre o nome da família da obra original e que dá título à obra, explorando o ruído emitido pelos gatos; temas como o incesto e o suicídio, poderosos catalisadores no processo narrativo, são ocultados ou sobrevalorizados pelo facto de que estamos perante textos com dimensões muito diferentes, sendo o texto original muito mais extenso do que o resultante da adaptação.

- $\quad$ Em T3, os grupos envolvidos na Reconquista Cristã correspondem a diferentes espécies de insectos com a respectiva adaptação do nome do povo correspondente sendo os Visigrilos centrais; há aqui, uma colagem evidente a recentes produções de animação, de ampla divulgação. Da mesma forma, as implicações políticas, o contexto religioso são ignorados, naquilo que de mais problemático e relevante apresentam, centrando-se o texto numa adaptação algo inconsistente dos atribulados amores de Eurico e Hermengarda.

- $\quad$ Em T4, no sermão, poderosa estratégia simbólica, originalmente destinado aos peixes, inverte-se a situação. É agora o peixe, quem recebe as crianças chegadas em visitas de estudo ao Fluviário de Mora, que tenta fazer um discurso moralizador referente à preservação do planeta e à luta contra a poluição. O local da pregação consiste num dos recentemente mais visitados aquários de água doce (Fluviário de Mora), caracterizado por recriar o universo aquático, envolvendo uma assumida vertente educativa e ambiental.

- $\quad$ Em T2, a original estrutura da aparição de tipos sociais no texto vicentino, recoloca os actores num palco que reduz a humanidade à escala da escola; as barcas são o prémio ou o castigo para os alunos tipificados em função de representações do senso comum e correspondendo a uma visão maniqueísta e estereotipada do que poderia ser uma comunidade escolar. O 'parvo', personagem de fronteira e de elevada liberdade no uso de um certo tipo de léxico aqui visto como reprovável, é ignorado. 


\section{Algumas reflexões finais}

Gostaríamos de deixar algumas reflexões finais que poderão, mais uma vez, ser apresentadas sob a forma de questões. Em que medida podem estes livros ser vistos como uma mais-valia no plano pedagógico, sobretudo no plano mais alargado de uma educação literária das crianças, uma vez que estas são o alvo explícito destes textos? Ultrapassando o entendimento de que uma reescrita não será mais do que outro texto e nunca mais o original, as estratégias de adaptação utilizadas são eficazes? Esta aproximação aos ‘clássicos’ implica que as crianças terão, em alguma medida, acesso aos textos originais ou estarão então elas a ler algo de completamente diverso que poderá funcionar como um filtro bloqueador de um posterior contacto com textos que poderiam ser interessantes, no sentido apontado por Calvino (2000).

A pressa e o entendimento falacioso de que este tipo de texto poderá facilitar o contacto com os textos originais ou poderá tornar mais fácil essa leitura posterior poderá correr o sério risco de colocar os leitores numa rota de leituras que, centradas em textos estranhos porque demasiado colados aos originais sem, contudo, se assumirem como uma paráfrase fiel, poderão ir além desta pseudo-estratégia de antecipação com benefícios ao nível de um rendimento escolar concebido como algo de muito restrito. Não consistirá esta experiência num programa irreversível de privação dos futuros leitores do contacto com os textos originais, sendo essa experiência substituída pelo contacto com realidades fragmentadas e distorcidas de um texto original perdido entretanto neste exercício comercial? Se a literatura na escola não ultrapassa, muitas vezes, a dimensão de uma abordagem colada a modelos teóricos rodeada de amplo conhecimento factual associado ao texto mas situado fora dele, que contributo poderão ter este tipo de reescritas na promoção da leitura como vivência dos textos, para além desse conhecimento institucionalizado, padronizado? Poderá a leitura de certos textos estar condenada à reprodução e validação dentro e fora da escola, de leituras alheias? 


\section{BIBLIOGRAFÍA}

- Altieri, Charles (1990). Canons and Consequences. Reflections on the Ethical Force of Imaginative Ideals. Evanston, IL: Northwestern University Press.

- Bourdieu, P. (1993). The Field of Cultural Production. New York: Columbia University Press.

- Calvino, I. (2000). Why read the classics. London: Vintage.

- Cassany, D. (2009). Para ser letrados. Voces y miradas sobre la lectura. Barcelona: Paidós.

- Chartier, A. \& Hébrard, J. (200o). La lectura de un siglo a outro. Discursos sobre la lectura (1980-2000). Barcelona: Editorial Gedisa.

- Culler, J. (2000). Literary Theory. A Very Short Introduction. Oxford: Oxford University Press.

- Dionísio, M. (200o). A construção escolar de comunidades de leitores. Leituras do manual de Português. Coimbra: Almedina.

- During, S. (200o). Teaching Culture. In David Richter (ed.) Falling into Theory. Conflicting Views on Reading Literature. New York: Bedford/St. Martin's.

-Eagleton, T. (2003). Literary Theory. An Introduction. Minneapolis: The University of Minnesota Press.

- Gorak, J. (200I). Canon versus Culture: Reflections on the Current Debate. New Cork: Garland Publishing.

- Lleal, J. (2OIO) . (ed.) Dialogant amb els clàssics : disset lectures del cànon literari. Girona : Universitat de Girona.

- Machado, J. (1990). Dicionário Etimológico da Língua Portuguesa. Lisboa: Livros Horizonte.

- Martins, M. (2003). Em teoria (A Literatura) In theory (Literature). Porto: Ambar.

- Said, E. (i994). Culture and Imperialism. New York: Vintage Books. 
- Said, E. (2000). The Politics of Knowledge. In David Richter (ed.) Falling into Theory. Conflicting Views on Reading Literature. New York: Bedford/St. Martin’s.

- Smith, B. (2000). Contingencies of Value. In David Richter (ed.) Falling into Theory. Conflicting Views on Reading Literature. New York: Bedford/St. Martin’s.

- Steiner, G. (2007). O siléncio dos livros. Lisboa: Gradiva. 